\title{
Development of driving cycles and user acceptance of pedelecs in rental systems: results of the electromobility showcase project pedelecs for recreation and tourism
}

\author{
S. Hölzel, A. Bedrunka, O. Benner, S. F. Andres, \\ L.-O. Gusig \& H. Hepp \\ Hanover University of Applied Sciences and Arts, Germany
}

\begin{abstract}
The research project pedelecs for recreation and tourism, a project of the showcase e-mobility, deals with the implementation of a manual pedelec rental system (60 pedelecs and 3 stations) in the city of Hanover (Germany). In addition to the 60 pedelecs, the Hanover University of Applied Sciences and Arts has also acquired 10 pedelecs, which the members of the University can use for free. This project investigates the pedelec requirements that are necessary for rental use in urban areas. As a result, pedelec dimensioning can be improved regarding costefficiency and robustness. Besides determining the technical requirements, the interest for electromobility should increase. The strategy is to identify measures for a proper rental concept in order to increase the acceptance of the population and contribute to an active use of this system. All pedelecs are equipped with a data logger, which records technical and GPS data. The technical data is used to determine the mechanical stress of the pedelecs while the GPS data is analyzed to identify the most frequently used routes. As a result of the outcome the Hanover driving cycle has been derived. Therefore, future operators and municipalities are able to acquire pedelecs based on the demands in Hanover. User acceptance is determined with surveys. The evaluation shows that there is a good basis for pedelec rental systems in Hanover, since people have a huge interest in riding a pedelec. Moreover, the university demonstrates that pedelecs are used by people of all ages. The study also shows the great potential for pedelecs, both for rental
\end{abstract}


systems and also for the retail market. With these vehicles it might be possible to achieve some of the desired $\mathrm{CO}_{2}$ reduction in urban areas.

Keywords: driving cycles, pedelecs, rental systems, user acceptance.

\section{Introduction}

The Hanover University of Applied Sciences and Arts has taken over the accompanying research in the project pedelecs for recreation and tourism at the electromobility showcase. In this project, a manual pedelec rental system has been built in the state capital Hanover and the district Goslar. A total of more than 110 pedelecs have been used.

The investigations of Hanover include the entire urban catchment of the project and therefore include the urban area (state capital of Hanover) as well as the tourist highland (district Goslar). The usage requirements and the resulting differentiating factor are the basis of these investigations.

For the general evaluation, a special data logger that records driving data and GPS data from the pedelecs had to be developed in the first place. The development was performed by the project partners Keymile and INSIDE M2M. In addition, the communication and database structure was built, which records and provides the receiving data of the pedelecs at any time. The data logger is installed directly onto the pedelec so that the communication concept shown in figure 1 is realized. The collected data of the logger forms the basis for the development of the Hanover-driving cycle.

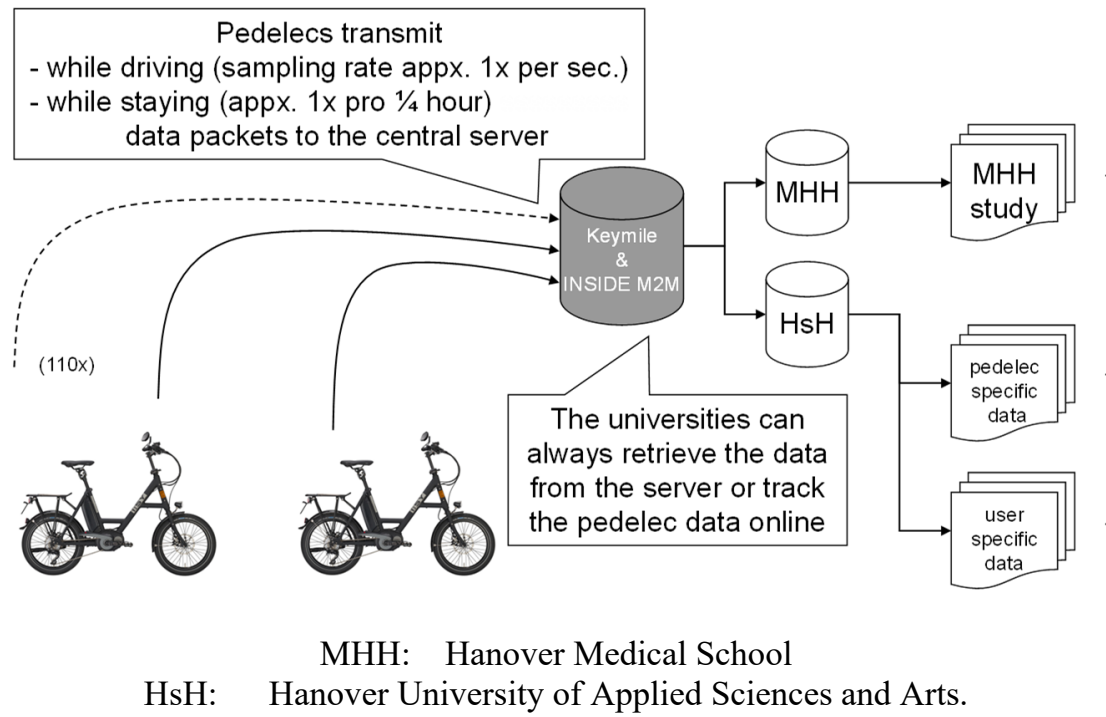

Figure 1: Communication concept of Hanover. 
Apart from the technical aspects, the acceptance of technological innovations is necessary for the development and establishment of new business models for pedelec rental systems. A successful project development can only be achieved by customer value and marketability.

The essential part of these investigations is the development of appropriate and customized questionnaires, the execution corresponding to surveys and the analysis of the completed questionnaires.

The effects and the optimization of appropriate rental systems are worked out by the collected data. The surveys also allow conclusions to the regular use of pedelecs in everyday life.

\section{Development of the Hanover driving cycle}

In order to investigate the technical requirements for rental use in urban areas, it is necessary to determine the strain on the pedelecs. Therefore, the average driving behavior of the population of Hanover has to be established, which can be achieved with driving cycles.

\subsection{State of the art}

In the automotive industry, standardized driving cycles are commonly used to determine the driving range and to evaluate emission behavior as well as energy efficiency of vehicles. For this purpose, a time-velocity-profile is being used which can either be produced synthetically or results from a real measurement [1]. Framework conditions for the determination of these values are defined in the regulation no.101 of the United Nations Economic Commission for Europe.

However, there are no specifications for determining the driving range in field of pedelecs. It is up to the manufactures to decide, under which circumstances the range would be evaluated [2]. Due to their interest in achieving the longest possible range, most of them normally test under ideal and therefore, unrealistic conditions (e.g. a constant velocity, lack of wind and gradient and a technically flawless pedelec). Thereby, it is impossible to compare different pedelec types or to rely on the manufacturer specifications regarding the range, which can lead to a lower acceptance among customers.

Regarding the pedelecs used in rental systems, driving cycles are needed in order to adjust the electronic to the driving behavior of the customer and therefore, reduce the default rate of the pedelecs.

\subsection{Methodological approach}

An approach to developing driving cycles for pedelecs is described in [3]. Based on this method, a model has been developed at the Hanover University of Applied Science and Arts with the software MATLAB, whereby it is possible to read in and evaluate a multitude of recorded driving profiles.

The analysis of the velocity profiles is performed by dividing them into microtrips. A microtrip is defined as the velocity profile between two breakpoints $(\mathrm{v}=0)$, which in turn is being analyzed regarding its average velocity and idle time 
and assigned to one of the groups "slow", "moderate" or "fast". Then for each group the total average is being calculated.

Subsequently the duration of the proposed driving cycle has to be set. The time should not be set too high, because examination on test benches, for example, would be economically inefficient. However, if the time is set too low, it might not be representative enough. Depending on the added up duration of every microtrip in a group the time slice of this group on the final driving cycle can be calculated. For example, if the time percentage of one group is $40 \%$, then it corresponds to the share of the group to the driving cycle.

Lastly, the microtrips which are most consistent with the group average regarding velocity and idle time are being put together for the final driving cycle.

\subsection{Hanover driving cycle}

With this approach, the Hanover driving cycle has been developed, where the database used for creating such a driving cycle has been recorded with the pedelecs in Hanover (figure 2). As described above, the driving cycle is divided into three parts. It takes exactly $861 \mathrm{~s}$ with an average speed of $18.1 \mathrm{~km} / \mathrm{h}$.

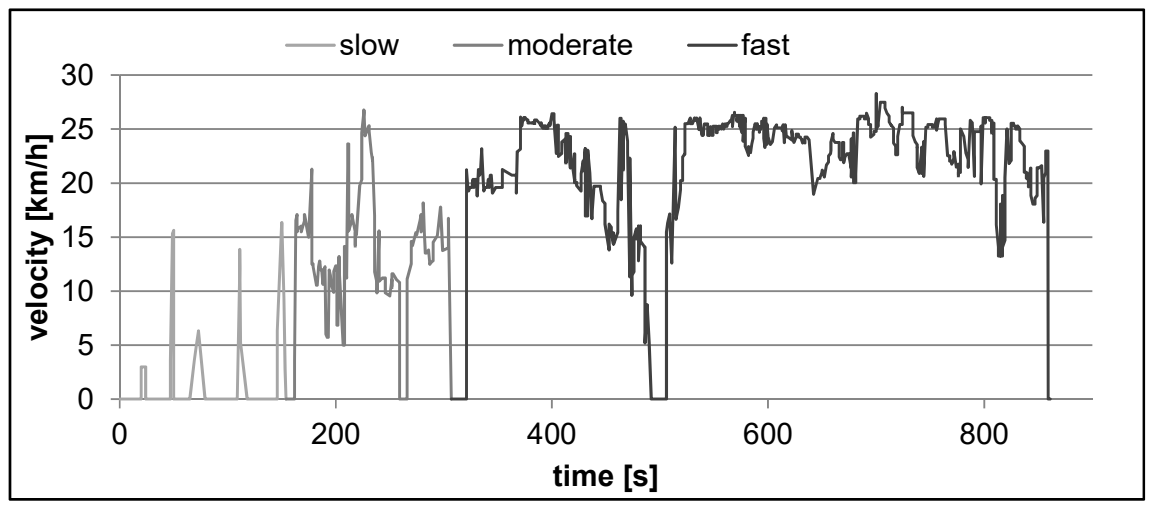

Figure 2: The Hanover driving cycle.

With the driving cycle it is possible to derive a stress profile for the pedelecs in Hanover. For this purpose, a simulation model is being developed at the moment. The simulation model is based on different test types which have been performed on the university's pedelec test benches. As a result of the simulation, pedelec dimensioning can be improved regarding cost-efficiency and robustness.

\section{User acceptance in rental systems}

Pedelecs are largely unknown to the population. There is only insufficient information available to convince users on a large scale. This is why the associations are unilateral to pedelecs and refer only to a few professional and age groups. 


\subsection{Method}

Survey techniques, methods and processes have been set within the investigation of the user acceptance. Therefore, the structure and content of the questionnaires of the given issues had to be derived for the user acceptance analysis.

The questionnaires presented certain participants' observations. The users have been interviewed regarding age group, gender, marital status, household income group, employment status, nationality and location as well as mobility patterns, travelled distance, reason for the trips, settlement of rentals, opinion/assessment of vehicles and technology. The developed questionnaires resulted by the phases of the research process by Raithel [4]. The developed phases for this project are shown in figure 3.

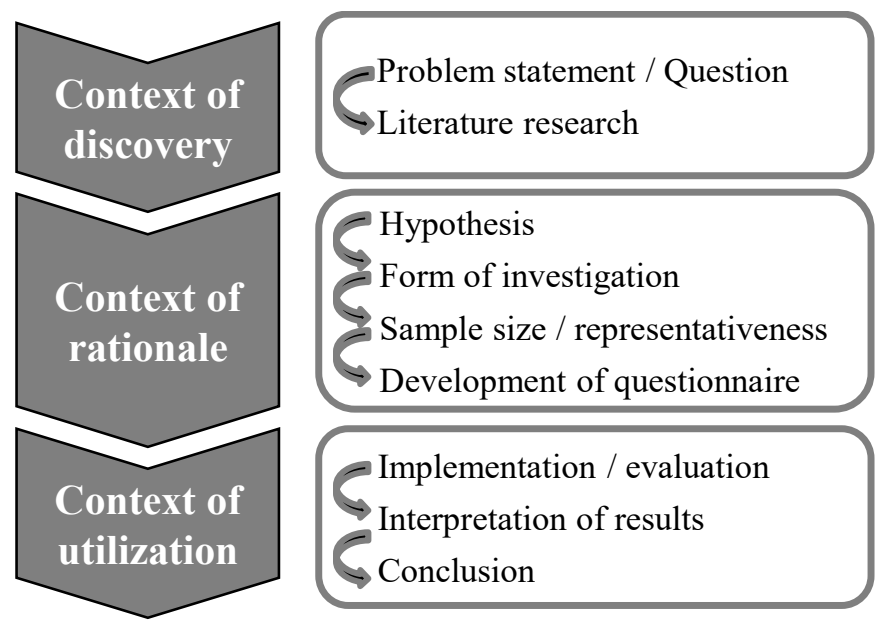

Figure 3: Evaluation phase cycle.

The written surveys of potential users were also supported by local oral interviews at fairs or at central locations in the cities of Hanover and Goslar.

In addition to the active survey, users were observed even passively by means of the GPS data from the data logger on pedelecs itself. In this case, the subject of investigation is the driving goals of the users as well as the frequency of use.

Moreover, bicycle and pedelec dealers as well as operators were interviewed alongside with the users. During these expert interviews data were collected regarding prospects and problems with pedelec rental from a commercial point of view.

\subsection{Representativeness of the results}

Due to the reconstruction of a pedelec rental system in Hanover and the new conditions attached, it is necessary to get a corresponding method to determine a representative size. A suitable method is the "home-use test", which served almost 
all requirements of the Hanoverian system. An essential feature is the review of a finished product by a representative sample of consumers. The product - here the pedelec - can be tested in a familiar environment and over a longer period. The group of subjects comprised about 200 to 400 people [5].

In addition to the users, bicycle and pedelec dealers as well as operators were also interviewed. In these expert interviews data were collected regarding prospects and problems with pedelec rental from a commercial point of view.

The operating strategy in Goslar differs from Hanover. While the customer in Goslar has to pay for the pedelec per day the use of the pedelecs in Hanover can be rented for free for a longer period of time (one day to three weeks). With the help of home-use tests it can be ensured to get representative information despite these difficulties.

The real sample sizes exceeding the determined target sample sizes. Therefore, it can be rated as representative (see table 1).

Table 1: Comparison nominal/actual sample sizes.

\begin{tabular}{|l|l|l|l|}
\hline $\begin{array}{l}\mathrm{Nr} \\
\text {. }\end{array}$ & Examination forms & $\begin{array}{l}\text { Specified - } \\
\sum \text { questionnaire }\end{array}$ & $\begin{array}{l}\text { Actual - } \\
\sum \text { questionnaire }\end{array}$ \\
\hline \multicolumn{3}{|c|}{ HANOVER } \\
\hline 1. & Oral survey: IdeenExpo & 1500 & 4658 \\
\hline 2. & Oral survey: passersby & $384^{*}$ & 433 \\
\hline 3. & $\begin{array}{l}\text { Participating observation }- \\
\text { pedelec user }\end{array}$ & $200^{* *}$ & 212 \\
\hline \multicolumn{3}{|l|}{ HARZ } \\
\hline 4. & Oral survey: Passanten & $383^{*}$ & 679 \\
\hline $\mathbf{5 .}$ & $\begin{array}{l}\text { Participating observation }- \\
\text { pedelec user }\end{array}$ & $148^{*}$ & 164 \\
\hline
\end{tabular}

*Sample size calculator:

http://www.bauinfoconsult.de/Stichproben_Rechner.html

**Home-Use-Test.

\subsection{Results of the user acceptance analysis}

The survey of potential users has shown large deficits in the level of awareness of pedelecs by people who have never tested these vehicles. This also had an impact on the overall image or reputation of pedelecs in society.

The term "pedelec" is not familiar to a majority of the population (55\%). Largescale publicity/promotions enable it to overcome this deficit and thus creating a significantly improved starting situation for a pedelec rental system. The surveys of potential users found, that about $55 \%$ of the interviewed passers-by do not know the term pedelec and its difference to an electric bike. $40 \%$ of the population cannot judge or exploit the available information on pedelecs or keep them quite inadequate.

With regard to a possible use of pedelecs, it is noted that a majority of respondents $(56 \%)$ ranked Pedelecs as a vehicle for the elderly or sick people. 
Surveys of users who could participate in trials as part of promotional events found, that this group has a different understanding about pedelecs. Approximately $90 \%$ of the group can imagine using a pedelec by themselves. Trying a pedelec improves enthusiasm as well as interest in pedelecs and reaches new target groups.

$33 \%$ would want to replace their cars with a pedelec. An essential impact on the modal split can occur by obtaining corresponding large target groups.

\section{The introduction of electromobility is important ("Yes")}

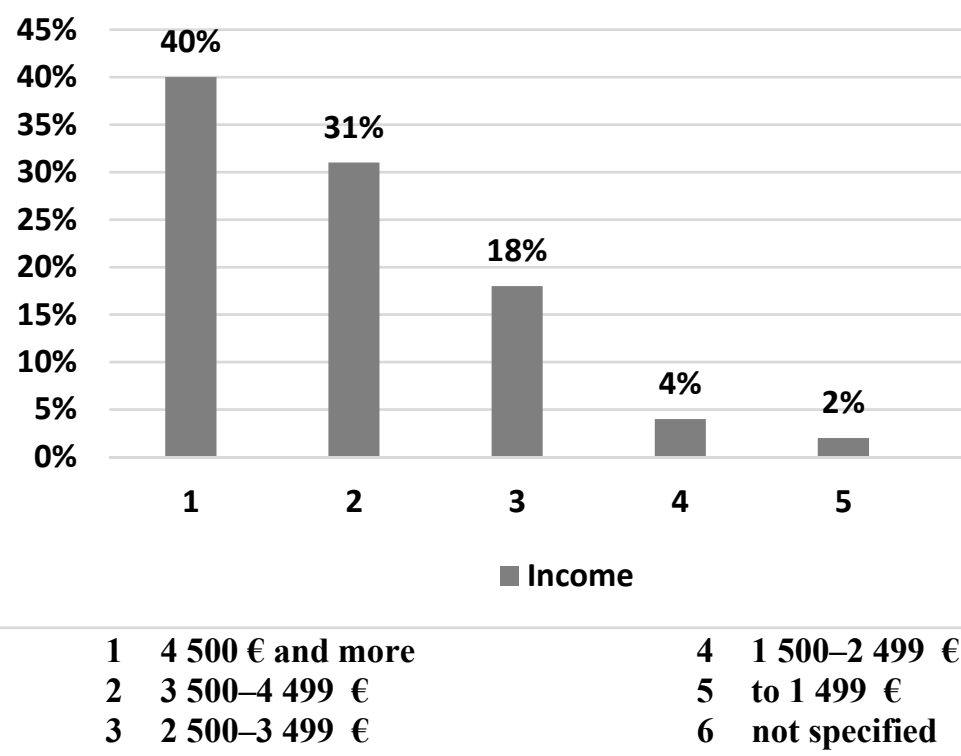

Figure 4: Connection between technology openness and income (net).

The resulting surveys included the issue regarding the reputation of pedelecs. The results show that pedelecs are generally considered to be environmentally friendly vehicles. For the users the driving comfort by the pedelec motor is not only the decisive factor. Moreover, Pedelecs are considered as a trend of the future in the population.

The openness to the technology and the associated higher prices rises with income. The higher the income of the people, the more they are open to the new technologies and the more they are interested in it (see figure 4).

\section{Outlook}

In future research, it will be necessary to develop a simulation model in order to derive stress profiles based on the Hanover driving cycle. On this basis, the calculation of the load of different pedelec parts can be performed. In this way, 
the dimensioning of the pedelecs can be executed with a focus on cost-efficiency and robustness.

Despite all the enthusiasm of users, it is necessary to initiate large-scale, targeted marketing measures to inform the population about two-wheeled electric vehicles and to raise their interest for the technology. Target group-oriented information materials and early provision of information are just two essential components of effective marketing measures for the electromobility. Pedelec rental systems cannot work profitably without these activities.

\section{Conclusion}

For an optimal cost-effective and robust dimensioning of the pedelecs in rental systems it is important to determine the strain on the pedelecs. To do so, first the driving behavior of the customers has to be determined which can be achieved with driving cycles. A suitable method has been developed with whom the Hanover driving cycle has been created.

The central challenge for the operation of pedelec rental systems is the low level of awareness of these vehicles. The awareness for target groups can be increased by appropriate marketing measures and campaigns. However, it cannot be done by individual companies because the financial and personnel cost would be too high. Central campaigns that have to be supported or carried out by public institutions are required.

\section{References}

[1] Ahmed, S. R. \& Hucho, W.-H. Aerodynamik des Automobils, Strömungsmechanik, Wärmetechnik, Fahrdynamik, Komfort mit 49 Tabellen. 6th ed., p. 138, Wiesbaden, Ger, 2013.

[2] greenfinder, Welche Reichweite hat ein E-Bike?. Retrieved 08.05.2015 from, http://www.greenfinder.de/news/show/welche-reichweite-hat-ein-e-bike/

[3] Bedrunka, A. \& Gusig, L.-O. Multidimensional parameter space for an individual prediction of the range in the field of pedelecs. In: Brebbia, C. A. (Hrsg.): Sustainable Development 2015, pp. 901-909.

[4] Raithel, J. Phasen des Forschungsprozesses im Überblick. VS Verlag für Sozialwissenschaften, p. 30, 2006.

[5] GPM Deutsche Gesellschaft für Projektmanagement e.V. Handbuch Produktmanagement: Strategieentwicklung - Produktplanung. p. 425, 2015. 